\title{
Melting of hard cubes
}

\author{
E. A. Jaglati \\ Centro Atómico Bariloche \\ Comisión Nacional de Energía Atómica \\ 8400 S. C. de Bariloche, Río Negro, Argentina
}

\begin{abstract}
The melting transition of a system of hard cubes is studied numerically both in the case of freely rotating cubes and when there is a fixed orientation of the particles - parallel cubes. It is shown that freelly rotating cubes melt through a first-order transition, whereas parallel cubes have a continuous transition in which positional order is lost but bond-orientational order remains finite. This is interpreted in terms of a defect-mediated theory of melting
\end{abstract}

\section{INTRODUCTION}

Freezing is probably the most unavoidable phase transition of a classical system of identical particles, occurring when temperature is reduced sufficiently. The most important difference between the low temperature (solid) phase and the high temperature (fluid) phase is that in the solid phase long range correlations between the coordinates of particles exist, whereas in the fluid phase this correlation is short range, decaying exponentially with distance. Freezing (or melting) is -for three dimensional systems- a first-order transition, with discontinuities in the first derivatives of thermodynamic potentials at the transition point. Compared to the liquid-gas transition or magnetic transitions in model systems, the advance in the theoretical understanding of the melting transition has been considerably slow.

Historically, there have been two main theoretical approaches to the problem of melting. Perturbative arguments 1 starting from the solid phase are able to predict that the solid structure will be unstable when temperature is increased sufficiently, but cannot automatically predict the characteristics of the fluid phase. Descriptions starting from the fluid phase (virial expansions, Ornstein-Zernicke equations, etc.), although give very accurate descriptions of the fluid phase in some model systems, usually do not predict at all a transition to a solid phase. The most successful account of the melting transition arising from this line of thinking is an order parameter theory of meltingt in which the most stable structure at a given temperature is obtained by minimizing a free energy functional which depends on the intensity of the Bragg peaks of the structure. These intensities are zero in the fluid phase, and finite in the solid phase.

A more recent approach to the problem is the theory of defect-mediated melting transition originally proposed for two-dimensional (2D) systems. The original idea is the following. 3 . The melting from the solid to the fluid phase is a consequence of the proliferation of defects in the perfect crystalline structure that exists at zero temperature. These defects, called dislocations, appear in pairs defect-antidefect, and have a finite binding energy.
As long as the size of the pair is finite, the positional order of the system is only perturbed at small length scales. When temperature is increased beyond a critical value $T_{m}$, the size of the pair becomes infinite, or in other words, free dislocations can exist in thermal equilibrium in the system. The existence of free dislocations disrupts the long range positional order of the system, and drives the melting transition of the crystal. The transition if predicted to be continuous, of Kosterlitz-Thouless type.t It was further realized that a crystal with a finite concentration of free dislocations does not behave exactly as a usual fluid. In fact, dislocations destroy the positional order of crystals, but not the orientational order that can still be present. In this scenario, the state of the system for $T>T_{m}$ corresponds to a fluid with orientational order, known as the hexatic phase. Within the hexatic phase a new kind of defects appear that destroy the orientational order at high enough temperatures. These defects were called disclinations by Halperin and Nelson. At a certain temperature $T_{o}$ disclination pairs unbind, and the system transforms in a usual fluid through a new Kosterlitz-Thouless transition. The critical values $T_{o}$ and $T_{m}$ depend on the self energies and interaction energies of dislocations and disclinations, which in turn are dependent on the parameters of the model. It should be noted that a necessary condition for this two-step melting process is that $T_{o}>T_{m}$, since orientational order can exist in the absence of positional order, but positional order cannot exist in the absence of orientational order. If the coupling between dislocations and disclinations is strong, the two continuous transitions can merge into a single firstorder transition. work was since then devoted to the confirmation of this theory of two-dimensional melting (that is known as the Kosterlitz-Thouless-Halperin-Nelson-Young -KTHNYtheory) and it was found in fact that the melting transition in two dimensions may be a single first order, 8 or a two-step continuous transition 9 depending on the system studied.

Due to its usefulness in 2D systems, it is tempting to apply the defect-mediated theory of melting to threedimensional (3D) systems. 6 In three dimensions, disloca- 
tions are one dimensional defects that form closed loops, or open lines that begin and end at the surfaces of the sample. In a 3D solid at low temperatures only small dislocation loops exist, but beyond a critical temperature infinitely large loops are present at equilibrium and the solid looses its positional long-range order. This picture of the transition is closely related to the superconductingnormal transition driven by the proliferation of vortex loops in superconductors, 10 or the superfluid-normal liquid transition in $\mathrm{He}^{4} .11$ Again, the transition driven by dislocations alone is continuous and the fluid above melting still has orientational long range order that generates a residual resistance to torsion, not present in a normal fluid 12 In analogy to the situation for 2D systems, a new class of defects responsible for the disappearance of orientational order must be introduced, and a possible new transition at higher temperatures should be expected.

From this point of view, the striking feature of the melting of identical particles in three dimensions is that it is always a first-order transition. This may indicate that the disclination-unbinding and dislocationunbinding transitions in three dimensions are strongly coupled, in such a way that they promote each other and make the transition first-order, but why this is always so is not known. The existence of a model system that melts through a continuous transition into a fluid with orientational order would be of importance in giving insight into the KTHNY theory in three dimensions.

The aim of this work is to analyze numerically a simple model which displays a continuous melting transition in three dimensions. The model is a system of impenetrable cubes, which have fixed orientation in space, the same for all cubes (parallel hard cubes, PHC) 13 Kirkpatrick 14 showed that this model has a continuous transition to a simple hypercubic solid structure in infinite dimensions, and suggested that this would also be so in three dimensions. The two main reasons to expect a continuous melting for PHC are the following. First, a cubic crystal lacks -in a Landau description of its melting - a third order term in the free energy functional that would favor the transition to be first-order 15 This kind of terms appear for crystalline structures that possess three Bragg vectors $\overrightarrow{G_{1}}, \overrightarrow{G_{2}}, \overrightarrow{G_{3}}$ lying on the first maximum of the diffraction pattern, and satisfying the relation $\overrightarrow{G_{1}}+\overrightarrow{G_{2}}+\overrightarrow{G_{3}}=0$. These vectors do not exist for a simple cubic structure. In addition, bond-orientational order 6 will be strongly enhanced in PHC compared for instance to spheres because a fixed orientation of each cube favors a neighborhood in which cubes arrange with the same orientation. This raises the possibility for the orientational order to persist up to higher temperatures than the translational order. For comparison, the case of freely rotating hard cubes (FRHC) will also be studied, and it will be shown that in this case the melting is a usual first-order transition into an isotropic fluid.

\section{NUMERICAL TECHNIQUE AND RESULTS}

The numerical method used to simulate the system is a standard Monte Carlo-Metropolis algorithm in the NPTensemble. The positions of the cubes are characterized by the coordinates of their centers. A trial movement of a particle consists of a displacement to a new position chosen randomly inside a cube of linear size $0.01 l$ centered at the old position ( $l$ is the linear size of the particles). The new position of the particle is accepted as long as there is no overlap with any other particle. After all particle coordinates are updated a trial global rescaling of all particle coordinates and system size by a factor within the range $1 \pm .01$ is proposed. If this change does not produce particle overlapping, then it is accepted according to the Metropolis algorithm with an energy change $d E$ given by $d E=P \Delta V-N k_{B} T \Delta V / V$ ( $N$ is the total number of cubes, $P$ is pressure and $V$ is the volume of the system). In the case of FRHC, in addition to the center-of-particle coordinates, the three Euler angles are necessary to characterize the position of each cube. These angles are updated at the same time as coordinates, the elemental change in each step is chosen to be $\sim 0.1$. Since there is no configurational contribution to the energy of the system, the equation of state depends only on the relation $T / P$. All results are presented as function of the adimensional temperature $T^{*} \equiv k_{B} v_{0}^{-1}(T / P)$ (that will be refered to simply as "the temperature"), where $v_{0}=l^{3}$ is the volume of each cube.17

The zero temperature state of the system of cubes (both parallel or freely rotating) is highly degenerate, because along any of the main crystalline directions, rows of cubes can be displaced an arbitrary amount without changing the volume of the system. However, at finite temperatures the cubic configuration with long range positional order has larger entropy than any row-displaced configuration, and the thermodynamically stable state is a simple cubic lattice.18 Even for the small systems that we are going to simulate, this entropy is greater than the one that can be gained by displacing rows of cubes (which is of the order of $\ln (N) / N$ ), and configurations with displaced rows never show up in the simulations in the temperature ranges of interest.

When temperature is increased sufficiently the crystal melts. This melting is qualitatively different for $\mathrm{PHC}$ and for FRHC. In the case of FRHC the melting occurs via a standard first order transition. Results of simulations are presented for a system of 125 particles. The system was initialized in a perfect cubic structure at low temperature, and a simulation was performed by increasing and then decreasing temperature. At each temperature 5000 Monte Carlo steps were used for thermalization and then 20000 steps were used to compute quantities of interest. In Fig. 1 (a) we see the evolution of the inverse packing fraction $v \equiv V /\left(N v_{0}\right)$ of the system. It shows a clear hysteretic behavior indicating a first-order phase transition 
for $T^{*} \sim 0.15$, where density changes between $\sim 0.45$ and $\sim 0.52$. Also shown in this figure as a dotted line are the values predicted from a cell-theory for the solid, 1 which gives a reasonable approximation to the real equation of state up to the melting temperature. It would be nice to have the expressions for the virial coefficients of FRHC to fit the fluid part of the curve, but these are not available to the required order to get a good fitting. For comparison, in Fig.1 the Carnahan-Starling equation of state of hard spherest is shown. The only free parameter is the sphere volume that was chosen to be $1.2 v_{0}$.

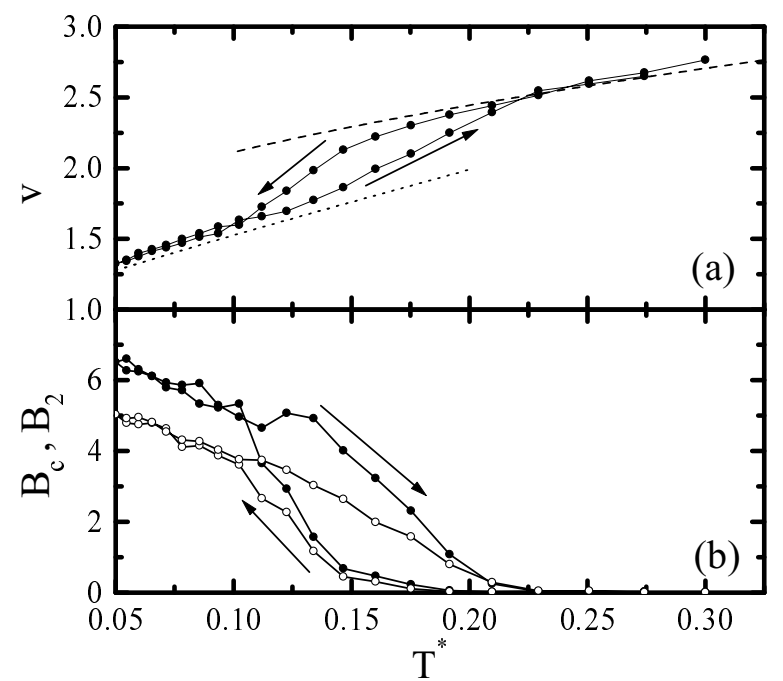

FIG. 1. Inverse packing fraction $v$ (a), Bragg intensities $B_{c}$ (b, full symbols) and nearest neighbors orientational order $B_{2}$ (b, open symbols) as a function of the adimensional temperature $T^{*}$ for a system of $5 \times 5 \times 5 \mathrm{FRHC}$, upon cooling (starting from a disordered configuration) and heating (see text for definition, $B_{2}$ and $B_{c}$ are given in arbitrary units). In (a) the dotted line is the prediction from a cell theory of the solid, and the dashed line is the behavior of hard spheres with an effective volume of $1.2 v_{0}$ in the fluid phase.

In Fig 1(b) two different indicators of the order in the system support the conclusion that the melting transition of FRHC is first order. The parameter $B_{c}$ is extracted from the diffraction pattern of the structure, and it is defined as

$$
\begin{aligned}
B_{c}= & \sum_{m=-4}^{4} \mid \int D(k, \theta, \varphi) Y_{4, m}(\theta, \varphi) \times \\
& \times\left.\delta\left(k-k_{1}\right) k^{2} d k \sin (\theta) d \theta d \varphi\right|^{2},
\end{aligned}
$$

where $D(k, \theta, \varphi)$ is the intensity of the diffraction pattern in polar coordinates, the delta factor picks up the values at the first maximum of the diffraction pattern $\left(k_{1}=2 \pi v^{1 / 3}\right)$, and the spherical harmonics $Y_{4, m}$ collect the part with cubic symmetry of the diffraction pattern. The value of $B_{c}$ is different from zero if the system possesses long range positional order. 19

The relative ordering of neighbor particles $B_{2}$ is defined as

$$
\begin{aligned}
& B_{2}=\sum_{m=-4}^{4} \mid \int D_{2}(r, \theta, \varphi) K(r) Y_{4, m}(\theta, \varphi) \times \\
& \times\left. r^{2} d r \sin (\theta) d \theta d \varphi\right|^{2}
\end{aligned}
$$

with $D_{2}(r, \theta, \varphi)$ being the pair distribution function of particles at distance $r$, along the spatial direction $(\theta, \varphi)$. The kernel $K(r)$ cuts off the integral beyond some distance. The results are qualitatively insensitive to the exact form of $K(r)$, in the results presented below $K(r)$ was taken to be 1 for $r<1.5 v^{1 / 3}$, and 0 for $r>1.5 v^{1 / 3}$. The value of $B_{2}$ is different from zero if the system possesses long range orientational order.

All these indicators of ordering vanish at the melting transition, with the same hysteretic behavior as that of the volume. The unambiguous determination of a firstorder phase transition would require the study of the volume histogram at the transition temperature, which should have a double peak structure associated to the coexistence of a solid and a fluid phase. Unfortunately, the simulation of FRHC is very time consuming so as to carry out this program. Partial checks were performed, however. In a simulation around the transition temperature $\left(T^{*}=.15\right)$ the volume of the system stabilized around different values, depending if the initial configuration of the system was chosen random or ordered. These values were the ones expected from Fig. 11(a). Partial simulations in systems up to 512 particles were performed, and the results are consistent with a first-order melting transition for FRHC.

If the cubes are restricted to be parallel to each other, the nature of the melting transition changes qualitatively. Results of simulations for this case are shown in Fig. 2 for a system of 216 particles. The volume of the system does not show any abrupt change, but a continuous and reversible (on heating and cooling) behavior. The parameter characterizing the crystalline order $B_{c}$ diminishes strongly around $T^{*}=0.4$, where the system has a density $\sim 0.5$, suggesting a continuous melting. The local orientational order (characterized by $B_{2}$ ), in spite of decreasing near the transition remains finite at high temperatures. This characteristic is not surprising since the orientational order is favored by the equal orientation of all cubes. In Fig. 2 we can see also the predictions for the volume from the lowest order cell model of the solid and the seventh order virial expansion for the fluid. 20 These expressions give a good approximation to the simulated values for all temperatures. 


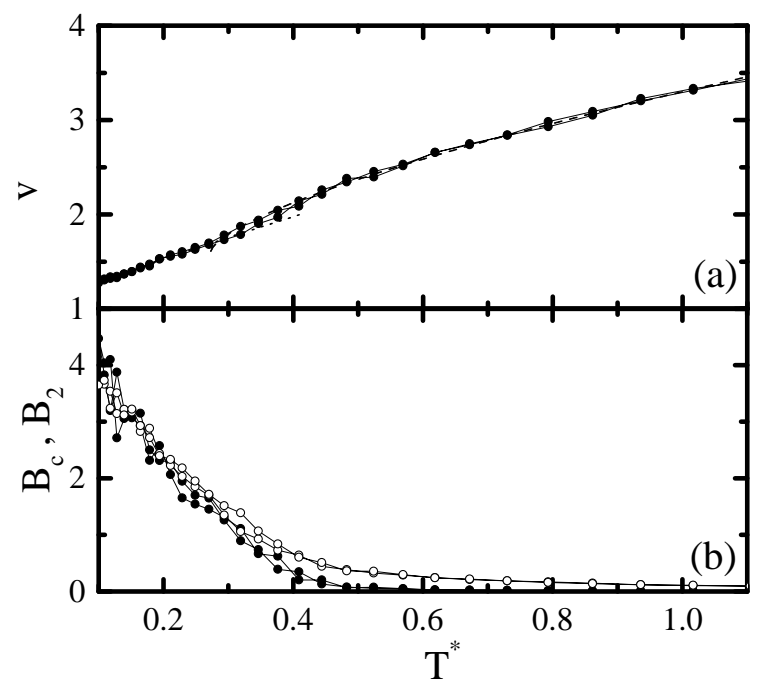

FIG. 2. Same as Fig.1 for a system of $6 \times 6 \times 6$ PHC. In (a) the dotted line is the prediction from a cell theory of the solid, and the dashed line is the equation of state for PHC to seventh order virial expansion.

If the melting of PHC is really a second order phase transition, the behavior of the order parameter of the transition (that can be taken to be the crystalline order parameter $B_{c}$ ) must obey scaling laws as a function of the system size. In particular, different simulations of $B_{c}$ in systems of different sizes $L\left(\equiv V^{1 / 3} / l\right)$ must obey a scaling relation of the form 21

$$
B_{c}=L^{-\mu} f\left(\left(T^{*}-T_{m}^{*}\right) L^{1 / \nu}\right),
$$

where $f$ is a universal function, $\nu$ and $\mu$ are two critical exponents and $T_{m}^{*}$ is the thermodynamical melting temperature. The exponent $\nu$ characterizes the divergence at the thermodynamic melting temperature $T_{m}^{*}$ of the correlation length. The result of simulations for systems of 216, 512, and 1000 particles are shown in Fig. 3. The volume and the orientational order $B_{2}$ show no detectable dependence on size, whereas the crystalline order $B_{c}$ has a clear size dependence. Results for $B_{c}$ for different system sizes can be collapsed reasonably well onto a single curve when plotted as $B_{c} L^{\mu}$ vs $\left(T^{*}-T_{m}^{*}\right) L^{1 / \nu}$, with parameters $T_{m}^{*}=0.40 \pm 0.02, \mu=4.0 \pm 0.5, \nu=0.50 \pm 0.05$. This value of $\nu$ is lower than the one corresponding to a three dimensional $X Y$ model, or the loop model for the normal-to-superconducting transition $(\nu=.666 \pm .003)$ that is supposed to be in the same universality class of our model if the melting can be described by the KTHNY theory. However, to be able to unambiguously decide this point, more simulations in larger systems are needed. The density of the system at melting is $0.48 \pm 0.02$.

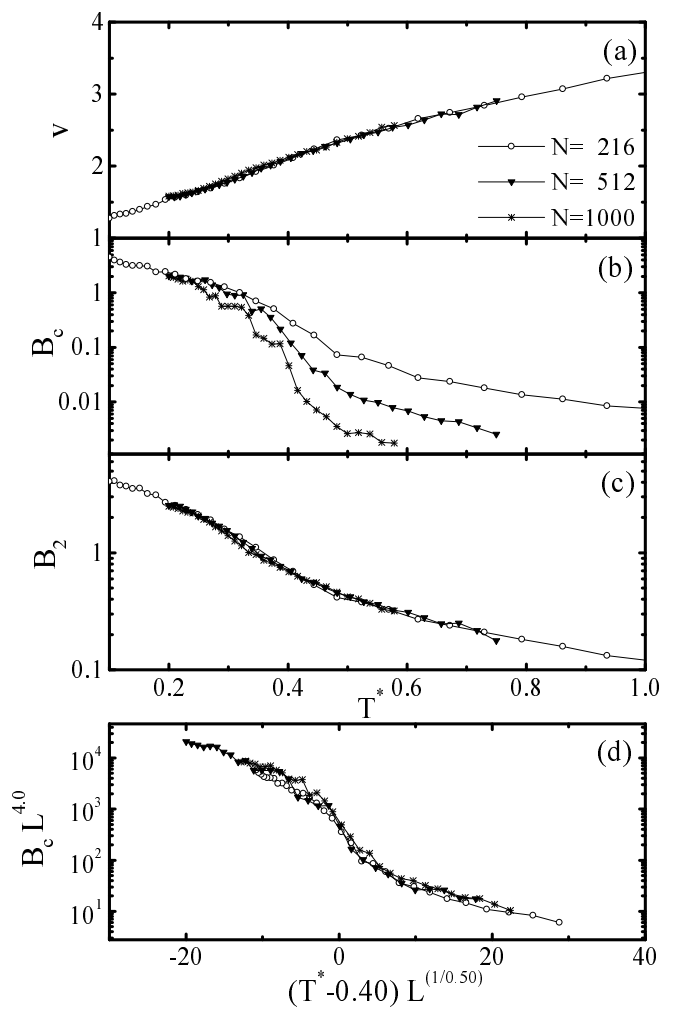

FIG. 3. Inverse packing fraction $v$ (a), orientational order $B_{2}$ (b), and crystalline order $B_{c}$ (c) as a function of temperature for systems of PHC of different sizes. The values shown correspond to an average upon cooling and heating. In (d) the curves of $B_{c}$ are scaled according to a second order phase transition using the adimensional linear size of the system $L \equiv V^{1 / 3} / l$.

The fluid formed by the parallel cubes above melting is not a usual isotropic fluid. This is obvious since some spatial orientations are singled out by the particular form of the particles. The fluid phase of PHC is the analogous of the hexatic phase of the KTHNY theory. Within this framework, the difference between FRHC and PHC is clear: parallel cubes keep the long range orientational order even when positional order has been lost, and the KTHNY theory predicts a continuous melting if only positional order is lost. FRHC have the possibility of loosing both positional and orientational order, and this is in fact what happens at a unique temperature in a discontinuous form.

It may be of interest to compare the fluid of $\mathrm{PHC}$ with the nematic phase of liquid crystals 22 In that case, molecules orient along a preferred spatial direction (i.e., they possess molecular-orientational order). Upon cooling, this structure transforms usually into a smectic-A phase in which a long range positional order is established along the direction characterizing the nematic phase. This transition may be first or second order depending on the material. At a lower temperature the smectic- 
A phase can undergo a transition to a crystalline phase. In our case, parallel cubes single out three orthogonal and equivalent directions in space, and upon cooling the system freezes into a solid phase, with crystalline order in all directions. The melting of PHC has no analogous in the transitions that occur in liquid crystal systems. Note that for the case of cubes the oriented phase has to be stabilized from outside, whereas the nematic phase in liquid crystals may be generated by molecular hard core interactions only.

\section{CONCLUSION}

In summary, I have shown numerical results on a simple model that displays a continuous melting transition in three dimension - namely a system of parallel hard cubes. The melting of this system can be qualitatively interpreted in terms of the KTHNY theory of defectmediated melting. The melting temperature was estimated to be $T_{m}^{*}=0.40 \pm 0.02$, and the critical density is $0.48 \pm 0.02$. The critical exponent of the correlation length is $\nu=0.50 \pm 0.05$. At the melting transition only positional order is lost, orientational order remains finite because it is favored by the geometric form of the particles. If the cubes are allowed to rotate, the melting is a usual first order transition where both positional and orientational order are lost.

\section{ACKNOWLEDGMENTS}

I thank K. Hallberg and D. Domínguez for critical reading of the manuscript. This work was financially supported by Consejo Nacional de Investigaciones Científicas y Técnicas, CONICET, Argentina.

$\dagger$ Electronic address: jagla@cab.cnea.edu.ar

${ }^{1}$ C. A. Croxton, Introduction to Liquid State Physics (Wiley \& Sons, Bristol, 1975); A. R. Ubbelohde, The Molten State of Matter (Wiley \& Sons, Bristol, 1978).

2 T. V. Ramakrishnam and M. Yussouff, Phys. Rev. B 19, 2775 (1979).

${ }^{3}$ B. I. Halperin and D. R. Nelson, Phys. Rev. Lett. 41, 121 (1978); A. P. Young Phys. Rev. B 19, 1855 (1979).

${ }^{4}$ J. M. Kosterlitz and D. J. Thouless, J. Phys. C 6, 1181 (1973).

${ }^{5}$ K. J. Strandburg, Rev. Mod. Phys. 59, 1001 (1987).

${ }^{6}$ D. R. Nelson, in Phase Transitions and Critical Phenomena, Vol. 7, Edited by C. Domb and J. L. Lebowitz (Academic Press, New York, 1983).

${ }^{7}$ H. Kleinert, Gauge Fields in Condensed Matter (World Scientific, Singapure, 1989).
${ }^{8}$ D. C. Glattli, E. Y. Andreim and F. I. B. Williams, Phys. Rev. Lett. 60,1710 (1988).

${ }^{9}$ A. J. Jin, M. R. Bjurstrom, and M. H. W. Chan, Phys. Rev Lett. 62, 1372 (1989).

${ }^{10}$ C. Dasgupta and B. I. Halperin Phys. Rev. Lett. 47, 1556 (1981).

${ }^{11}$ R. P. Feynman, Prog. Low temp. Phys. 1, Chap 2 (1955); V. N. Popov, Zh. Eksp. Teor. Fiz. 64, 672 (1973) [Sov. Phys. JETP 37, 341 (1973)].

12 D. R. Nelson and J. Toner, Phys. Rev. B 24, 363 (1981).

13 B. T. Geilikman, Proc. Acad. Sci. USSR 70, 25 (1950).The system of $\mathrm{PHC}$ has been used recently as a model for the study of demixing in fluid mixtures. See M. Dijkstra, D. Frenkel, and J. P. Hansen, J. Chem. Phys. 101, 3179 (1994); J. A. Cuesta, Phys. Rev. Lett. 76, 3742 (1996).

14 T. R. Kirkpatrick, J. Chem. Phys. 85, 3515 (1986).

${ }^{15}$ P. M. Chaikin and T. C. Lubensky, Principles of Condensed Matter Physics, (Cambridge University Press, Cambridge, 1995) Ch. 4.

${ }^{16}$ In the system of PHC, molecular-orientational order, i.e., order in the orientation of the individual particles always exist, since all particles are parallel to each other by definition. Bond-orientational order refers to long range correlations in the orientations of the segments joining the centers of neighbor particles. This is what is tested with the parameter $B_{2}$ (see text). I will use simply "orientational order" to refer to "bond-orientational order".

17 Simulations were also performed by fixing the volume of the system and calculating the equilibrium pressure (and thus $T^{*}$ ) along the simulations (the $N V T$-ensemble). The results are completely consistent with the ones presented here.

${ }^{18}$ In fact, the simple cubic structure of hard cubes has at low temperatures a shear modulus proportional to $T^{*}$ along the main crystalline directions (E. A. Jagla, unpublished).

19 Strictly speaking, long range orientational order alone generates values of $B_{c}$ which are different from zero. However, these values are much smaller than in the presence of long range positional order. This justifies to use $B_{c}$ as an indicator of long range positional order.

${ }^{20}$ W. G. Hoover and A. G. De Rocco, J. Chem. Phys. 36, 3141 (1962); W. G. Hoover and J. C. Poirier, J. Chem. Phys. 38, 327 (1963).

${ }^{21}$ D. Stauffer and A. Aharony, Introduction to Percolation Theory (2nd Ed, Taylor \& Francis, London, 1991).

22 S. Chandrasekhar, Liquid Crystals (Cambridge University Press, Cambridge, 1977). 